ARTICLE

DOI: $10.1038 / \mathrm{s} 41467-017-00542-3$

\title{
A DNA nanoscope via auto-cycling proximity recording
}

Thomas E. Schaus ${ }^{1,2}$, Sungwook Woo ${ }^{1,2}$, Feng Xuan ${ }^{1,2}, X_{i}$ Chen ${ }^{1,2}$ \& Peng Yin ${ }^{1,2}$

Analysis of the spatial arrangement of molecular features enables the engineering of synthetic nanostructures and the understanding of natural ones. The ability to acquire a comprehensive set of pairwise proximities between components would satisfy an increasing interest in investigating individual macromolecules and their interactions, but current biochemical techniques detect only a single proximity partner per probe. Here, we present a biochemical DNA nanoscopy method that records nanostructure features in situ and in detail for later readout. Based on a conceptually novel auto-cycling proximity recording (APR) mechanism, it continuously and repeatedly produces proximity records of any nearby pairs of DNA-barcoded probes, at physiological temperature, without altering the probes themselves. We demonstrate the production of dozens of records per probe, decode the spatial arrangements of 7 unique probes in a homogeneous sample, and repeatedly sample the same probes in different states.

\footnotetext{
${ }^{1}$ Wyss Institute for Biologically Inspired Engineering, Harvard University, 3 Blackfan Circle, 5th Floor, Boston, MA 02115, USA. ${ }^{2}$ Department of Systems Biology, Harvard Medical School, Boston, MA 02115, USA. Sungwook Woo and Feng Xuan contributed equally to this work. Correspondence and requests for materials should be addressed to P.Y. (email: py@hms.harvard.edu)
} 
S patial organization of molecular components is fundamental to the function of synthetic $c^{1-5}$ and biological ${ }^{6-8}$ nanostructures. This organization can be described as a set of pairwise proximities between the components. Single-molecule methods to study proximity and organization must examine individual nanostructures with molecular-scale precision, and are foundational to advancing nanoscience ${ }^{9-12}$

Though limited to multiplexing a modest number of simultaneous species, direct visualization by electron, atomic force, and optical microscopy have identified individual macromolecular associations in synthetic ${ }^{4,5,13}$ and biological ${ }^{14-16}$ systems, and achieved molecular resolution in controlled, static environments ${ }^{17}$. Resonance energy transfer (FRET) techniques further allow for dynamic measurement of pairwise proximity in solution $^{18,19}$. Complementary to microscopy are the biochemical techniques that have enabled ensemble measurements of protein interaction networks with molecular precision, including yeast two-hybrid ${ }^{20-22}$ and related ${ }^{23}$ assays, affinity purification/mass spectroscopy ${ }^{24}$, and co-immunoprecipitation ${ }^{25}$. They are relatively easily parallelized, executed, and even automated.

Barcoding with DNA has recently given biochemical techniques the potential for single-molecule precision. Proximity ligation assay (PLA) ${ }^{26}$ or proximity extension assay $(\mathrm{PEA})^{27}$, which record the colocalization of two probes by ligating or extending probe-bound DNA sequences, now routinely multiplex $\sim 100$ signals with orthogonal sequences. The information content of DNA itself is much larger, however. There are $4^{N}$ combinations of $N$ nucleotides, enabling 1 million-plex with only 10 -nucleotide strings. Unfortunately, because ligated or extended probes are depleted in detecting a single pairwise association, no more than a single association per molecular target can be recorded. This makes it difficult to reconstruct complexes on a singlemolecule basis.

Here, we present a new biochemical interrogation technique that records nanostructure features in situ and in detail for later readout. Termed auto-cycling proximity recording (APR), it repeatedly produces proximity records of any nearby pairs of DNA-barcoded probes, without altering the probes themselves. We describe and characterize the mechanism in detail, and then demonstrate a biochemical DNA nanoscope that decodes the complex spatial arrangements of seven unique APR probes in a homogeneous sample. We further show that different states of the same probe set can be repeatedly interrogated.

Although we used PCR and gel-based assays on homogeneous samples to demonstrate the nanoscope, APR can in principle be applied with uniquely barcoded probes and read with massively parallel sequencing. We expect that further development of smallsample sequencing pipelines ${ }^{28}$ and computational analysis will enable APR to achieve massively parallel, single-molecule precision.

\section{Results}

The APR concept. Figure 1a describes the inherent limitations of current, pairwise-destructive methods in the amount of information recorded from an individual structure. Because only a single proximity is recorded from each probe, proximity information remains isolated in unconnected pairs and reconstruction is incomplete. In contrast, APR (Fig. 1b) generates proximity data autonomously and repeatedly, at tunable distances, by nondestructively copying pairs of DNA-barcoded probes. The result is a complete set of proximity information and complete reconstruction. The APR mechanism also provides for high signal levels, as well as allows resampling of the same molecular targets in different proximity arrangements.
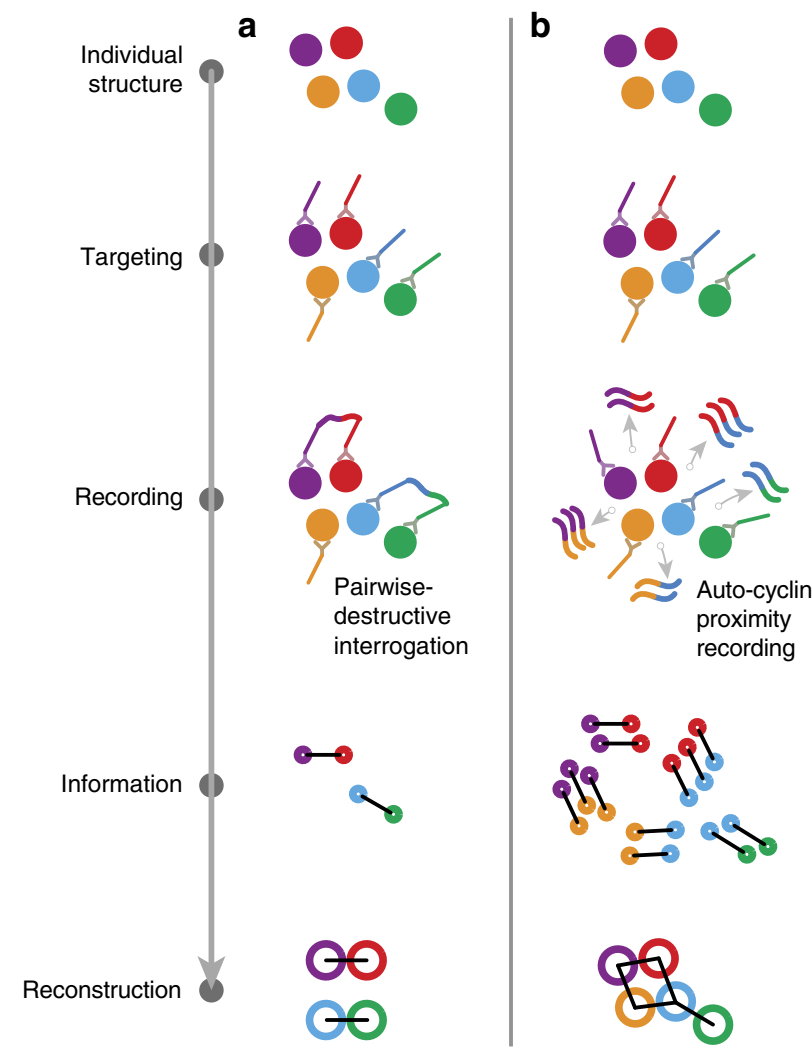

Fig. 1 Comparison between existing pairwise-destructive proximity measurements and auto-cycling proximity recording (APR). a In pairwisedestructive methods (e.g., proximity ligation), a particular target-bound probe generates a proximity record with only one neighbor that it becomes permanently attached to. This in turn yields only limited proximity information for a particular multitarget structure, necessarily resulting in incomplete reconstruction of its target arrangement. $\mathbf{b}$ With APR, proximity records are generated autonomously and continuously by transient pairing of any nearby probes, without destroying or depleting them. Not only are multiple copies of a record generated for a particular pair of probes, improving signal, but each probe can also produce proximity records with every neighbor, potentially allowing for complete reconstruction

The APR mechanism. The APR reaction proceeds in an autonomous, cyclic fashion, at physiologic conditions, to convert soluble primers into molecular records of probe pair proximity (Fig. 2a). As configured for ensemble applications, DNA hairpin probes $P_{\mathrm{i}}$ and $P_{\mathrm{j}}$ are first attached to target molecules $T_{\mathrm{i}}$ and $T_{\mathrm{j}}$ via antibodies, aptamers, direct nucleic acid hybridization, or other means, and encode the identity of the target type within unique primer binding sequences (labeled as sequence domains $a_{\mathrm{i}}$ and $a_{\mathrm{j}}$ ). In step (i) of Fig. 2a, the soluble primers (sequences $a_{\mathrm{i}}^{*}$ and $a_{j}^{*}$, where the asterisk denotes a complement) stably bind their respective hairpin probe type and are extended by a polymerase through the spacer $(s)$ and palindromic $(p)$ domains up to the stopper site, thereby producing Half-records $\left(a_{\mathrm{i}}^{*}-s^{*}-p^{*}\right.$ and $\left.a_{\mathrm{j}}^{*}-s^{*}-p^{*}\right)$. In step (ii), Half-records are partially displaced by spontaneously re-forming hairpin stems $\left(s^{*}-p^{*} / p-s\right)$ and (step iii) bind any nearby Half-records at their $3^{\prime}$ palindromic $\left(p^{*}\right)$ segments. These sequences are again extended in step (iv), this time over the partner spacer $s^{*}$ and primer $a^{*}$ segments, causing the release of Full-records that carry both probe identities as $a_{\mathrm{i}}^{*}$ and $a_{\mathrm{j}}^{*}$. Probes, thus regenerated to their initial state, may undergo additional cycles in the same or other pairings. Upon termination of this cyclic recording reaction, a specific Full-record species can be tested for by PCR 

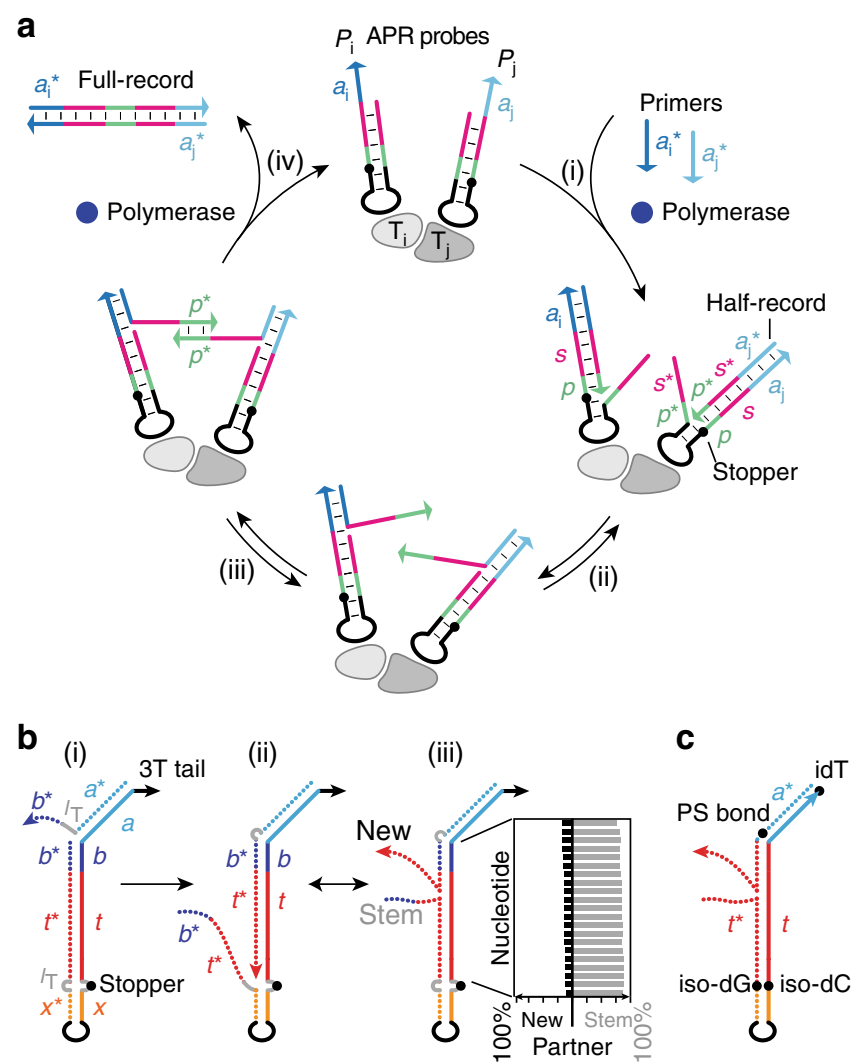

Fig. 2 Auto-cycling proximity recording (APR) mechanisms. a The APR cycle creates molecular records of proximity from pairs of hairpin probes. Probe-specific primers are (step $i$ in a) extended to Half-records and (ii) reversibly displaced, (iii) bind palindromic domains of nearby Halfrecords, and (iv) are extended on each other to create and release Fullrecords, regenerating the probes. See text for detailed description. b Copyand-release hairpin $(\mathrm{CRH})$ detail, depicting primer binding domain $b$ explicitly but labeling the entire copied template simply as $t$. Shown is the mechanism of (state $i$ in $\mathbf{b}$ ) initial primer binding, (ii) extension, and (iii) random walk of the strand displacement branch. For this combination of primer bulge $I_{T}$ and Spacer9 stopper shown, the hairpin template strand $t-b$ is computationally predicted to pair predominantly with the hairpin stem complement (iii, plot). See also Supplementary Fig. 1. c A more rapidly cycling hairpin does not utilize bulge $I_{\mathrm{T}}$ or domain $b$, but instead uses a phosphorothioate (PS) bond before the final primer ( $a$ ) nucleotide, in conjunction with synthetic nucleotide pair iso-dC/iso-dG as a stopper. See Supplementary Fig. 2 for sequences and Supplementary Note 1 for other considerations

amplification (with respective primer pair $a_{\mathrm{i}}^{*}, a_{\mathrm{j}}^{*}$ ) and gel electrophoresis. In this manner, for example, an isolated sample with PCR primers for $a_{\mathrm{i}}$ and $a_{\mathrm{j}}$ would yield a positive result in the presence of $T_{\mathrm{i}}-T_{\mathrm{j}}$ proximity.

This cycle solves two main challenges in generating multiple records between any nearby pairs. First, a template sequence (in Fig. $2 \mathrm{a}, s^{*}-p^{*}$ ) must be copied from the probe and then spontaneously made single stranded and available for further reaction. Autonomous cycling requires that this proceeds without external thermal or chemical influence. Second, in the context of target proximity, each pair of copied sequences must be combined into a single molecular record and then released.

The first challenge is solved by a DNA-based copy-and-release hairpin $(\mathrm{CRH})$ that enables the isothermal copying and release of an arbitrary sequence onto each primer strand (Fig. 2b). While simply extending a primer on a single-stranded template, as in PCR, would leave the product stably hybridized, here the template is bound to a complementary sequence that aids in its removal. The detailed mechanism for most experiments is as follows. In step (i), DNA primer strand $a^{*}-l_{\mathrm{T}}-b^{*}$ (i.e., of domains $a^{*}$ and $b^{*}$ with intervening single $\mathrm{T}$ nucleotide linker $l_{\mathrm{T}}$ ) binds the $3^{\prime}$ end of the hairpin at primer binding domain $a$. In step (ii), the primer and hairpin $b^{*}$ domains compete for short probe domain $b$. Primer domain $b^{*}$ occasionally binds, despite the resulting single $\mathrm{T}$ nucleotide $l_{\mathrm{T}}$ bulge, and is extended by a displacing polymerase (here, Bst, New England Biolabs, or Bsm, Thermo Fisher) through stem template domain $t$. When primer extension reaches a stopper modification near the loop (here, covalent triethylene glycol linker Spacer 9, Integrated DNA Technologies (IDT), opposite a single $\mathrm{T} l_{\mathrm{T}}$ ), the polymerase dissociates. In step (iii), the new extension and the displaced hairpin stem, being identical in sequence $\left(b^{*}-t^{*}\right)$, rapidly compete for stem template domains $t-b$ via the strand displacement mechanism ${ }^{29}$ with millisecond transit times ${ }^{30,31}$. Computer models $\left(\mathrm{NUPACK}^{32}\right.$ ) predict that the stem template $t-b$ is more likely to be bound to its stem-based complement than to the new primer extension (iii, plot, as well as Supplementary Fig. 1), ensuring the Halfrecord is largely single-stranded and therefore available for further reaction. In contrast, the $16 \mathrm{nt}$ length of sequence $a^{*}$ ensures that the Half-record remains stably bound until displaced by a polymerase.

The second APR design challenge, linking these Half-records to release a Full-Record in the context of proximity, is solved by copying a short, 6-nucleotide palindromic sequence $p$ (ACGCGT or similar in Fig. 2b, or stronger GGCGCC in Fig. 2c) to the $3^{\prime}$ end of the Half-record. Here, a palindrome is defined such that $p \equiv p^{*}$, enabling any Half-record to bind to any other Half-record by their $3^{\prime}$ ends. Upon hybridization of two $p$ sequences, extension by the polymerase copies the information of each Half-record onto the other, ultimately displacing and releasing the nascent Full-record from both probes. Note that nonspecific interaction between unbound Half-records is prevented by two features: the $p$ domain is not part of the soluble primer but only added upon Half-record formation on a probe, and these Half-records are only released once they pair. See Supplementary Fig. 2 for further probe detail and sequences.

Details of the CRH probe are important to reliability and cycling speed. Among the most important is the mechanism that biases the system toward a closed hairpin and single-stranded Half-record $\left(b^{*}-t^{*}\right)$, in which the cycle can progress. In Fig. $2 \mathrm{~b}$, depicting the probe design used in all figures except Fig. $3 b$, the stopper represents an internal loop ${ }^{33}$ in the hairpin duplex. Though the loop terminates polymerase extension, the one nucleotide $(\mathrm{T})$ tether between hairpin complement $t^{*}$ and lower stem $x^{*}$ slows initiation of the strand displacement process that releases the Half-record. Left unbalanced by a similar effect at the primer end, this would result in a predominantly open hairpin and bound Half-record $b^{*}-t^{*}$. The T nucleotide bulge ${ }^{33}$ within primer $a^{*}-l_{\mathrm{T}}-b^{*}$ effects this balance. It increases the overall rate of cycling despite slowing down initial primer extension.

Further development led to the improved probe described in Fig. 2c, which utilizes an unnatural nucleotide pair (iso-dC/iso$\mathrm{dG}, \mathrm{IDT}$ ) as a stopper. This tighter stopper does not require such a strong balancing weakness in the primer. A simple phosphorothioate bond between the last two primer nucleotides, with no $b^{*}$ domain, weakens hybridization enough to bias the system toward a closed hairpin. After optimizing the palindrome and conditions (see below and Methods), the cycling speed of this probe is $\sim 5$-fold faster than that of Fig. 2 b.

To minimize secondary structure and prevent spurious binding and extension of primer $a^{*}-b^{*}$ or extension $a^{*}-b^{*}-t^{*}$, these domains utilize only three base types ( $\mathrm{A}, \mathrm{T}$, and $\mathrm{C})$. The short palindrome domain $p$, a component of template $t$ in the APR 
cycle, is a necessary exception. See Supplementary Note 1 for other considerations.

Proof of principle. Figure 3 presents the proof-of-principle experiments, demonstrating proximity-based, auto-cycling, Full-record generation. Figure $3 \mathrm{a}$ focuses on the proximity dependence of reactions. Lanes 1-4 each contained combinations of two probe types, made different only in spacer length for the purpose of differentiating products on gel electrophoresis, together with a universal Cy5 fluorophore-labeled primer and a polymerase. In lane 1 , short and long barcode probes existed unbound in solution. The primers (red, in excess) were extended to short or long Half-records, respectively, but no Full-records were generated because probes were not in close proximity of each other. In lane 2 , short barcode probes were held in proximity on streptavidin molecules, and the system produced short Halfrecords and, in turn, short Full-records, as expected. When streptavidin was prepared in separate vessels with either short or long probes, and then mixed together for reaction (lane 3), both Half-records and Full-records of short and long lengths were generated. Interaction among probes from different streptavidin molecules would have also produced Full-records of intermediate length (i.e., of long+short Half-records), but these were instead only present when short and long probes were randomly arranged on the same streptavidin molecules, as in lane 4 .

Figure $3 \mathrm{~b}$ demonstrates the autonomous cycling ability of the probes by observing product formation over time. Because primers were each labeled with a single copy of Cy5, the amount of product in each band could be quantified. Beginning with a 1000 -fold abundance of primers $(100 \mathrm{nM})$ with respect to probes $(0.1 \mathrm{nM})$, probes free in solution generated undetectable levels of Full-record. When held together on streptavidin, however, each probe was utilized 30 times on average, with an initial record generation rate of $\sim 1 \mathrm{~min}^{-1}$.

Conclusive proof that a given individual probe sequentially generates records with multiple different partner probes was provided by massively parallel DNA sequencing. Probes with a universal primer domain and random stem domains (creating barcodes unique to each probe) were bound by streptavidin and recording was performed. Figure $3 c$ shows an example of a single probe (with Barcode $i$ ) generating records with three other probes (Barcodes $j^{*}$ ), as expected. See Supplementary Fig. 4 for mechanism and details.

Probe reach characterization. Probe design dictates the reach of a probe pair, or probe-probe proximity within which Full-records can be produced. To characterize the rates of Full-record production as a function of probe-probe distance, pairs of probes were fixed to programmed positions on two-dimensional (2D) DNA origami ${ }^{3}$ structures (Fig. 4a). Three types of probes were tested, encompassing three probe spacer lengths and two attachment methods. Two types were attached as extensions of intrinsic origami staple strands (Fig. 4a, inset (i)), while a third was held by a click-chemistry azide-alkyne linkage to an intermediate strand (inset (ii)) to demonstrate how probes may be attached to arbitrary moieties. Both methods incorporated single-stranded DNA as flexible linkers at the base of each probe. Figure $4 \mathrm{~b}$ diagrams the flexible, single-stranded and the rigid, double-stranded components contributing to the reach between two probes.

Recording reactions for each probe and separation distance were carried out separately. Many copies of a given origami structure were held flat and immobile on a mica surface, as is done for atomic force microscopy of DNA structures (see Supplementary Note 2 and Supplementary Fig. 7 for details
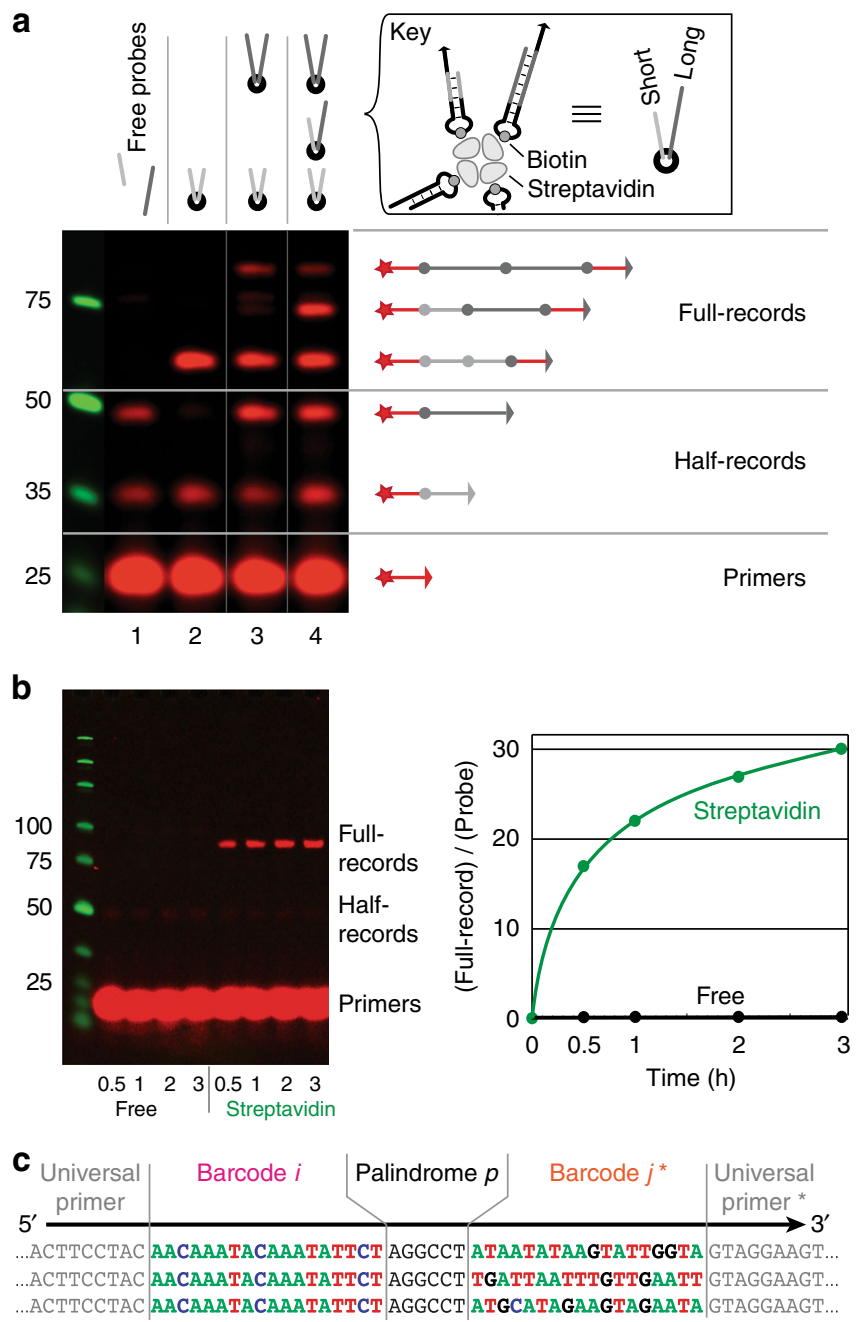

Fig. 3 Proof-of-principle experiments. a Generation of Full-records requires the colocalization of probes, here by biotinylated hairpin loops bound to streptavidin, whereas isolated probes can always generate Half-records. Cropped denaturing PAGE gel depicting $10 \mu \mathrm{l}$ reactions ( $40 \mathrm{~min}$ at $37^{\circ} \mathrm{C}$ ) with biotin-streptavidin association and 4:1 overall probe/streptavidin stoichiometry (inset), 8 and $22 \mathrm{nt}$ barcodes (19 and $33 \mathrm{nt}$ stem lengths copied), 10:1 initial primer/probe, and $40 \mathrm{nM}$ total probe concentration. A single primer sequence was used and no secondary amplification was performed. b Auto-cycling is demonstrated by quantification of Cy5-labeled probes on cropped denaturing PAGE gels. Rapidly cycling probes with Iso-dC/dG stoppers and phosphorothioate primers were used, with probes at $0.1 \mathrm{nM}$ and primers at 1000 -fold excess to probes in each time series. Quantification of Full-records yields the plot. Half-records are difficult to detect because of low probe concentration. See probe details and sequences for a, b in Supplementary Fig. 2 and full gels in Supplementary Fig. 3. c An example of a single probe (with Barcode $i$ ) making multiple partnerships (with Barcodes $j^{*}$ ), read with Illumina MiSeq next-generation sequencing. Here, probes encoded a universal primer sequence and unique barcodes (in place of spacer $s$ of Fig. 2a), and were held in tetramers by streptavidin. Primer sequences cropped for clarity. See Supplementary Fig. 4 for the unique-barcode APR cycle, probe details, and sequencing methods

of how this stabilizes structures against displacing polymerases). Records were then amplified by PCR, and products quantified by gel electrophoresis. To account for variation in experimental conditions, especially in the number of origami, a reference probe pair with orthogonal PCR sequences was also present on each origami type, but always at the same fixed separation distance 

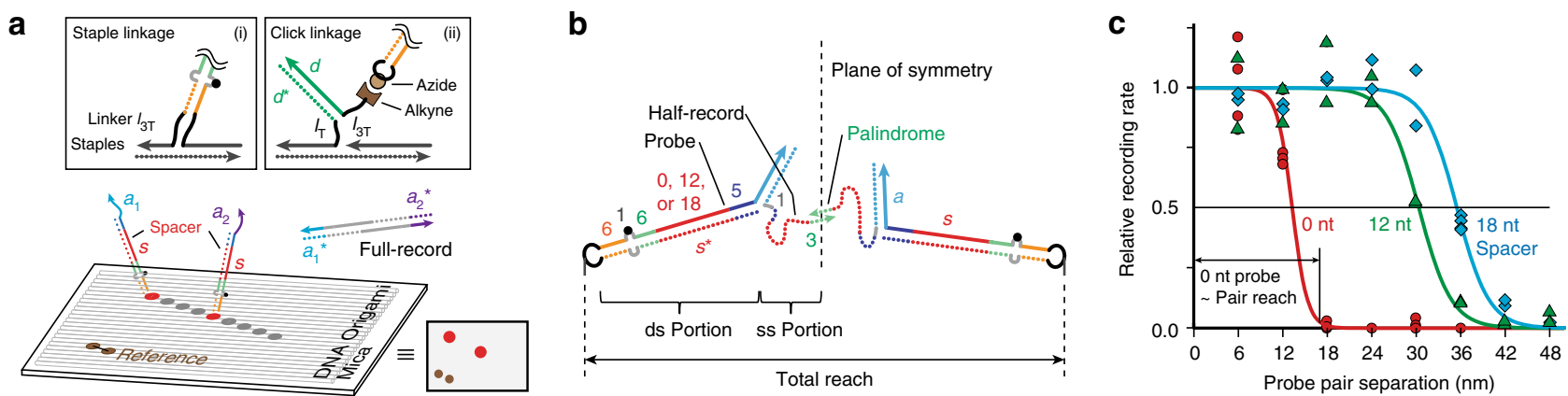

Fig. 4 Probe reach. a To characterize reach, relative probe positioning was controlled precisely. Probes were attached to 2D, rectangular, twist-corrected DNA origami structures measuring 70 by $100 \mathrm{~nm}$ (origami shown with all possible Fig. 4 probe positions, as well as equivalent cartoon with 4 probes; see Supplementary Fig. 5 for origami design, Supplementary Table 1 for staple sequences, and Supplementary Fig. 6 for precise probe positions). After assembly, origami were deposited randomly on mica surfaces in the presence of $12.5 \mathrm{mM} \mathrm{Mg}^{2+}$ for firm adhesion, a condition we find protects origami from degradation by polymerase (see Supplementary Note 2). Each probe was a direct extension of two origami staples (inset i) or covalent attachment to an intermediary oligonucleotide (inset ii; azide on probe loop covalently attached to alkyne on $I_{3 T^{-}} d$ intermediary via DIBO-based click chemistry and gelpurified). Separation distances were measured between origami attachment points. Records were collected from the supernatant without disturbing the underlying origami, and PCR-amplified with, for example, $a_{1}^{*}$ and $a_{2}^{*}$ primers. $\mathbf{b}$ The maximum reach across which a probe pair can make a Full-record is determined by the cumulative lengths of their components, including double-stranded probe components and single-stranded Half-record components. These are shown as rigid and flexible, respectively, corresponding to their persistence lengths of $\sim 50 \mathrm{~nm}^{38}$ and $\sim 2 \mathrm{~nm}^{39}$. Numbers indicate lengths in nucleotides. c Otherwise identical probes with spacer lengths of 0 or $12 \mathrm{nt}$ (attached by staple extension), or $18 \mathrm{nt}$ (covalently attached), were held in pairs separated by 6 to $48 \mathrm{~nm}$ in $6 \mathrm{~nm}$ increments (red positions in a), recorded ( $1 \mathrm{~h}$ at room temperature, $100 \mathrm{nM}$ primers), and log-phase PCR-amplified (20 cycles, $500 \mathrm{nM}$ primers) to gel-detectable levels. Denaturing PAGE band quantification was normalized to a constant reference pair for each well. Data for a given probe pair type were fit by least squares recursion to a simple sigmoidal curve $c_{1} /\left(1+\operatorname{Exp}\left[c_{2}\right.\right.$ (dist- $\left.\left.c_{3}\right)\right]$ ), where dist represents separation distance and $c_{1}$ through $c_{3}$ were fit, and normalized to a maximum rate of 1 . See probe sequences in Supplementary Table 2

(Fig. 4a). The rate of production was calculated in relation to this reference pair.

Figure $4 \mathrm{c}$ shows the relative recording rate for three probe designs, containing 0,12 , and $18 \mathrm{nt}$ spacer domains $s$. Each probe pair was tested every $6 \mathrm{~nm}$ for separations of 6 to $48 \mathrm{~nm}$, and mathematical fits to record generation rates were normalized to a common maximum. The 0,12 , and $18 \mathrm{nt}$ probes all produced records at near maximum rates when pairs were closest to one another. Rates were reduced to half at 13,20 , and $25 \mathrm{~nm}$, respectively, and had a maximum reach ( $1 \%$ of maximum recording rate) at 17,39 , and $44 \mathrm{~nm}$. In absolute terms, the $0 \mathrm{nt}$ spacer probe produced records at the slowest rate, approximately half as fast as longer probes.

All three maximum-reach distances correspond to those expected when DNA probes and attached Half-records are oriented in a straight chain (Fig. 4b). For the 0 nt spacer probe, the maximum expected distance is approximately twice the sum of the probe length $(19 \mathrm{bp}$ of double-stranded DNA, at $0.34 \mathrm{~nm}$ per base pair ${ }^{34}, \sim 6.5 \mathrm{~nm}$ ) and Half-record length (11 nt of singlestranded DNA minus 3 nt palindrome overlap, at the maximum $0.58 \mathrm{~nm}$ per base ${ }^{34}, \sim 4.6 \mathrm{~nm}$ ), totaling $\sim 22 \mathrm{~nm}$. Similarly, the 12 and $18 \mathrm{nt}$ spacer probes have a maximum reach of $\sim 44$ and $\sim 55 \mathrm{~nm}$, respectively, at which recording has stopped. See Supplementary Note 3 and Supplementary Fig. 8 for estimates of reach profiles using worm-like chain models.

Interrogation of complex geometries. APR can be used to gently interrogate extensive proximity relationships within a homogeneous sample of structures, enabling reconstruction of overall geometry from PCR-based assays (Fig. 5). The longest probe tested in Fig. 4c, with an $18 \mathrm{nt}$ spacer (s), was first used to determine the relative proximities of three targets. Three probes, programmed with different primer binding sequences $(a)$ and therefore generating records with unique ends (e.g., ends $a_{\mathrm{i}}^{*}$ and $a_{j}$ in Fig. $2 \mathrm{a}$ ), were again fixed by $2 \mathrm{D}$ origami in a triangular configuration with $30 \mathrm{~nm}$ probe separation (Fig. 5a). This placed each probe within the reach of the other two. A recording was performed, as before, and the sample was split into three volumes, reflecting the number of potential pairwise probe combinations. Each subsample was combined with a different pair of PCR primers, and records, if any, were amplified. Gel electrophoresis and band quantification indicated if records were produced, and a graph depicting relative positioning was computationally reconstructed. As expected, the triangular arrangement yielded all three record types, suggesting that very geometry. In contrast, when the same probes were placed in a straight line (Fig. 5b), only two record types were produced, as the distance between P1 and P2 was beyond probe reach.

More complex, 7-point geometries were then tested, each using shorter, $12 \mathrm{nt}$ spacer $(s)$ probes arranged on a smaller, $24 \mathrm{~nm}$ hexagonal pattern (Fig. $5 \mathrm{c}-\mathrm{e}$ ). This made nonadjacent probes at least $42 \mathrm{~nm}$ apart, beyond the reach of these probe pairs $(\lesssim 39 \mathrm{~nm})$. Each 7-probe geometry required testing of $21(6+5+4+3+2+1)$ possible probe pairings, yielding one of $2^{21}>2 \times 10^{6}$ possible binary combinations, any potentially valid. As in Fig. 5a, records were produced from these origami, split, and amplified by PCR in the 21 possible primer pairings. The hexagonal geometry of Fig. $5 \mathrm{c}$ yielded 12 of 21 positive proximity pairs, as seen by gel electrophoresis, in addition to the 21 reference pairs that served as positive PCR controls. Simple graphing of these 12 pairs (Wolfram Mathematica, GraphPlot function, see Supplementary Note 4) resulted in the correct geometry.

The exceptional fidelity of reconstruction in this case is due to a combination of experimental and reconstruction details. The graphing algorithm used here is based on a physical model (spring-electrical) that separates the final probe positions relatively uniformly, and is therefore particularly congruent with the regular hexagonal organization of the origami probes. A sense of the precision of this reconstruction is shown in Fig. 5c, inset (i). Given the positions shown for any six probes, the seventh probe may be anywhere within the shaded region and still satisfy the gel data shown. In addition, a series of graphs 


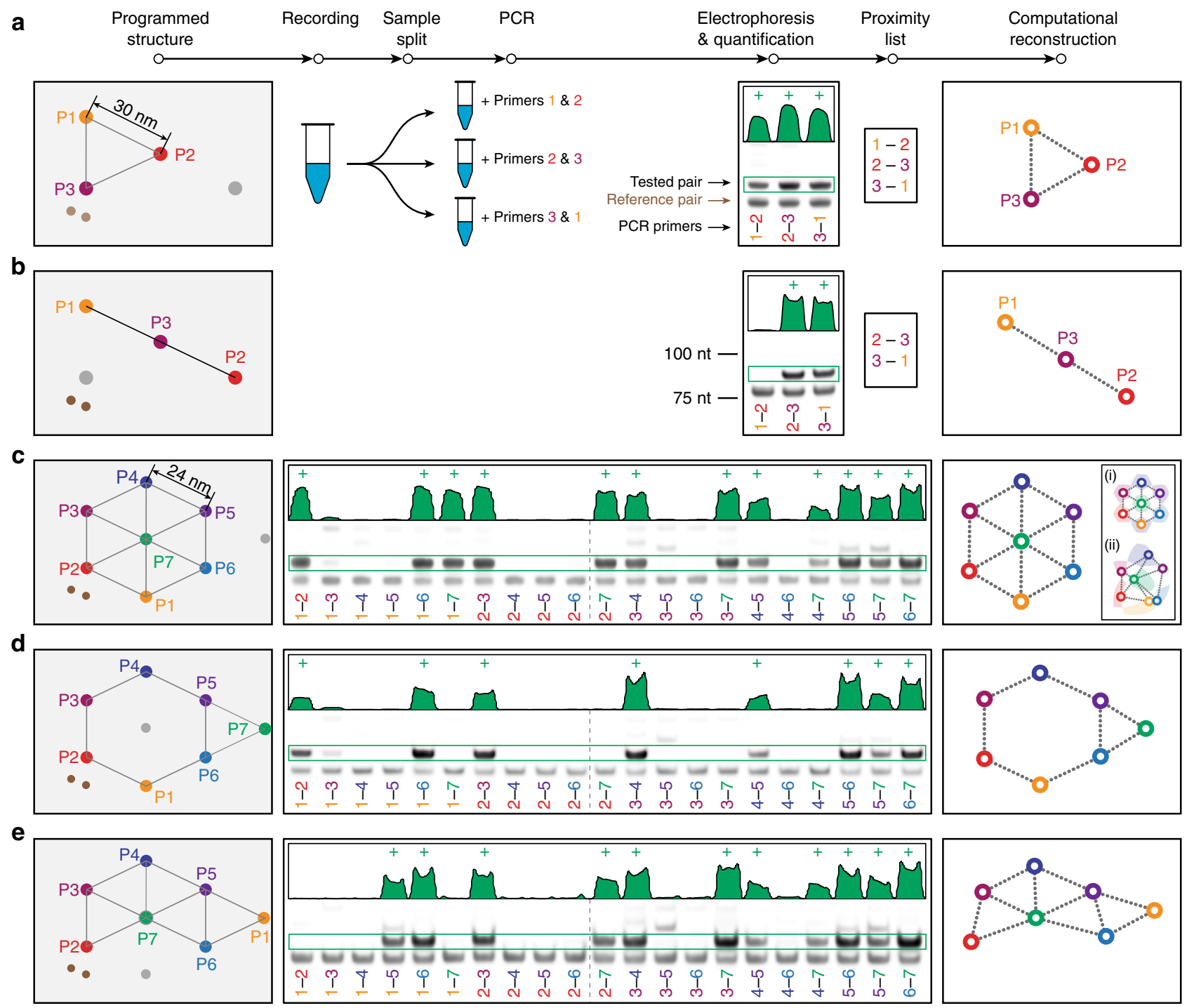

Fig. 5 Complex geometry interrogation. a The same rectangular origami base used in Fig. $4 \mathrm{~b}$ was adapted to hold three probes, of $18 \mathrm{nt}$ spacer design, at $30 \mathrm{~nm}$ spacing (see Supplementary Fig. 6). In the same conditions as in Fig. 4, probes were first placed in a triangular arrangement and recording was carried out. The sample was split, records PCR-amplified with separate pairs of probe primers (and reference primers), and products separated by gel electrophoresis and quantified (green plot profiles, marked (+) when judged to qualify for the proximity list). As expected, all three probe pairs yielded records, suggesting a triangular shape in reconstruction. $\mathbf{b}$ When those same probes were attached in a line, with P1 and P2 out of reach from one another, only two record types were amplified. This suggests a relatively linear arrangement. c More complex, 7-probe geometries were also interrogated, this time with $24 \mathrm{~nm}$ hexagonal grid spacing and $12 \mathrm{nt}$ spacer probes. The full set of 21 primer pair amplifications yielded the gel shown, with 12 pairs in proximity. Computational reconstruction yielded the same hexagonal pattern. Insets show positional precision of any single point, with respect to others shown, as shaded region that satisfies this gel. Inset (i) shows the precision for computed hex pattern, while inset (ii) shows the precision for a random pattern that also satisfies gel result. d When center probe P7 was moved outside the hexagon, APR yielded the 8 expected proximities and correct reconstruction.

e Similarly, when P1 was moved, the 11 expected proximities and correct reconstruction resulted. Throughout, computational reconstruction images were reflected and rotated, but not distorted, for best comparison to the programmed structure diagrams. See probe sequences in Supplementary Table 2 and full gels in Supplementary Fig. 9. All reference bands in the figure correspond to lengths indicated in $\mathbf{b}$

with random probe positions were generated and tested for compatibility with these same data. One compatible example is shown in the Fig. 5c inset (ii), again with shaded regions showing the precision of a given probe position. See Supplementary Fig. 10 for more examples.

Two further examples are demonstrated. Figure $5 d$, where the center probe was moved to the outside, yielded the eight correct proximity pairings and geometry. Figure $5 \mathrm{e}$, where the lower probe was moved to the outside, yielded the 11 correct proximities and geometry.

Note that the relative quantity of Full-records created should not be taken as a measure of relative distance between probes, but only that the appearance of records indicates that probes were within reach (proximity); the nonlinear record generation rate as a function of distance (Fig. 4c), variable probe attachment to target, and possible sequence dependence make distance approximation from precise record count unreliable. Instead, further refinement of target-target distances may be performed by sequential experiments with probes of different reach. For example, a pair of $12 \mathrm{nt}$ spacer probes may produce records, indicating targets closer than $36 \mathrm{~nm}$, while a pair of $0 \mathrm{nt}$ spacer probes at those same positions do not, indicating those same targets are also farther than $18 \mathrm{~nm}$. Alternatively, a single experiment with a mixture of probes with unique primers and 


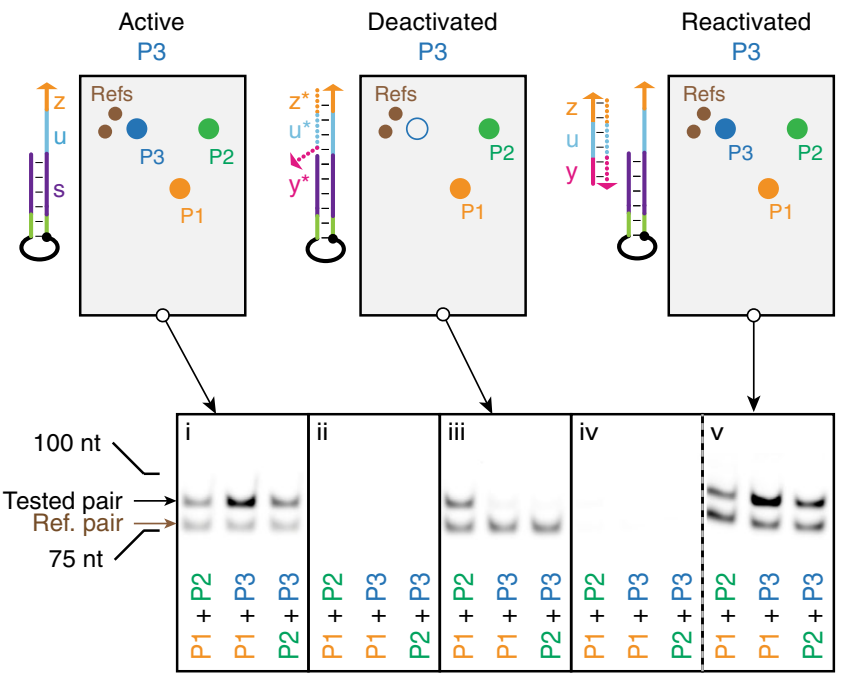

Fig. 6 State change. The same origami probe triangle was used as in Fig. 5a, but probe P3 could be deactivated. (see Supplementary Fig. 6 for precise probe positions.) PCR amplification and denaturing PAGE of recording from active probe (i), after intermediate wash (ii), from deactivated probe (iii), after second intermediate wash (iv), and from reactivated probe (v). The intermediate wash results demonstrate that there were no leftover records. A single set of origami was used for all steps and resampled. Origami and PAGE otherwise treated as in Fig. 5. See probe sequences in Supplementary Table 2 and full gels in Supplementary Fig. 12

variable reach could be used. See Supplementary Fig. 11 for examples of how reconstruction precision could be further refined with this strategy.

Resampling of a state-changing system. An advantage of nondestructive recording is that the same probes can be repeatedly sampled, even in a system undergoing changes in its state. The origami-based triangular arrangement of probes in Fig. 5a was again constructed (Fig. 6), with a mechanism for inactivating probe $\mathrm{P} 3$ by shielding its primer binding site $(u-z)$ with a blocker strand $\left(z^{*}-u^{*}-y^{*}\right)$, as well as for subsequently reactivating P3 by removing the blocker with strand $y-u-z$. As such, the same tri-target system can be made to cycle between two distinct states, with P3 initially active (stage i, Fig. 6), then inactive (stage iii), and finally active again (stage v).

We applied APR to interrogate this state-changing system. At each sampling point, the supernatant was split, PCR amplified, and observed by gel electrophoresis. First, the triangular arrangement was recorded (with primer $u^{*}$ on P3) and analyzed, indicating the colocalization of three probes as expected (Fig. 6, i). After a wash with buffer, the supernatant showed no residual records (Fig. 6, ii). Then, by applying inextensible (inverted dT at $3^{\prime}$ end to prevent extension) P3 primer blocker $z^{*}-u^{*}-y^{*}$, any leftover $\mathrm{P} 3$ primers and Half-records were displaced and probe $\mathrm{P} 3$ was made unable to bind primer, rendering it deactivated. Recording then indicated colocalization of only P1 and P2 (Fig. 6, iii). To sample a third state, the same origami were again washed and shown record-free (Fig. 6, iv). Probe P3 was then reactivated by addition of strand $y-u-z$, which removed the primer blocker. Recording and analysis once again indicated the full triangular arrangement (Fig. 6,v), completing three correct samplings of the same targets.

\section{Discussion}

Given that structure dictates function in synthetic and natural nanostructures, we sought to record nanoscale proximity and organization in as precise and complete manner as possible. To this end, we have developed a biochemical technique termed APR. The two key mechanisms requiring development were a nondestructive, continuous (i.e., auto-cycling) method for copying DNA strands from probes, and a method for pairing these strands only in the presence of probe proximity (proximity recording). The first was solved by the development of the CRH (also recently suggested elsewhere ${ }^{35}$ ), which allows a probe-specific primer to be extended into a closed hairpin using the energy in deoxynucleoside triphosphates (dNTPs), and then isothermally releases the template by re-closing the hairpin via the strand displacement process. The second, proximity requirement was enforced by encoding a short palindromic site at the $3^{\prime}$ end of the extension. The extended primers represent Half-records that must pair by their palindromes with other nearby Half-records before they can extend over each other, through their primer domains, and be released into solution as Full-records of proximity. Subsequent identification of these record sequences reveals the pattern of proximity.

The APR method shares a superficial resemblance to PLA and PEA techniques in that all three record pairwise interactions in DNA. A key difference is that because APR probes are not consumed in detecting each proximity, many pairwise recordings reflecting the underlying equilibrium conformation can be made from each probe. We have taken advantage of this to demonstrate the detection and reconstruction of complex geometries and the resampling of the same molecular system in different states, all with simple PCR and gel-based readouts. The geometry reconstructions placed 7 probes in the correct relative position, a 1 -in- $2 \times 10^{6}$ event by chance alone. Resampling allowed the single sample to be recorded in three different arrangements. Neither, to our knowledge, have been demonstrated with proximity ligation or other biochemical techniques. While pairwise ligation of many copies of a target might be expected to yield the same geometry results, in our own previous attempts ligation was often detected between probes that were implausibly far apart. We hypothesize that ligation operates as a latch that permanently connects even rare pairings distorted under thermal motion. These improper ligations distort the underlying geometry and make further pairings inaccurate as well. Conversely, APR makes only transient connections between probes, allowing the underlying proximities to reflect the desired equilibrium state.

No ensemble experiment detecting pairwise proximities can differentiate between a complex structure and separate pairings that overlap to form that structure. Because APR generates $\sim 30$ records per probe, providing sensitivity to most or all neighboring connections, it suggests the challenging but exciting prospect of single-molecule analysis. Random probe barcodes could be used to uniquely identify each of, for example, $10^{6}$ molecular targets, and massively parallel next-generation sequencing could read out every recorded proximity on a per-molecule basis. Specificity against chance (non-repeating) interactions could be enforced by neglecting any proximity read found only once ${ }^{36}$. Such a single-molecule capacity may enable, for example, an assay that counts any number of multiplexed protein types in cell lysates (by applying antibodies to two separate epitopes of each protein type), measurement of molecular interaction stoichiometry and turnover, and of course singlemolecule network connectivity that differentiates complexes from separate components. Alternatively, non sequencing-dependent detection of a soluble, low-concentration protein could suppress false positives by requiring two interactions between the same probe pair to create a single record. We are actively pursuing several of these capabilities. 


\section{Methods}

Probes. Probes were ordered from IDT with possible modifications of internal biotin (/iBiodT/), internal azide (/iAzideN/), internal Spacer 9 (/iSp9/), internal iso-dC/dG pairs (/iMe-isodC/ and /iisodG/), or phosphorothioate bonds (denoted by “*” between nucleotides). See Supplementary Fig. 2 and Supplementary Tables 1 and 2 for probe sequences. Strands were ordered at 100 or $250 \mathrm{nmol} \mathrm{scale}$, with high-performance liquid chromatography (HPLC) purification.

Some probes were ordered with an internal biotin modification in the center of the $5 \mathrm{nt}$ loop domain, in which case probes were held in proximity with streptavidin, as indicated in Fig. 3 and Supplementary Fig. 2. Probes were attached to streptavidin (New England Biolabs (NEB)) in a $20 \mu$ reaction containing $25 \mathrm{nM}$ streptavidin (NEB) and $140 \mathrm{nM}$ probe (for a total of more than $4: 1$ probe/ streptavidin to ensure streptavidin saturation), held at $37^{\circ} \mathrm{C}$ for $1 \mathrm{~h}$.

Other probes were covalently linked to alkyne-modified strands by click chemistry. Azide-modified raw probes were ordered directly from IDT and dibenzocyclooctyne (DBCO) group modified linker strands were purchased from Boston Open Labs. Both DNA strands were HPLC purified by the manufacturers. A volume of $9 \mu \mathrm{l}$ of $1 \mathrm{mM}$ DBCO-linker strands and $1 \mu \mathrm{l}$ of $10 \times$ phosphatebuffered saline ( $\mathrm{pH} 7.4$ ) buffer were added to $20 \mu \mathrm{l}$ of water containing $180 \mathrm{pmol}$ azide-modified probes and incubated at room temperature for $12 \mathrm{~h}$. The linked probes were purified by $8 \%$ denaturing polyacrylamide gel and quantified by NanoDrop UV-Vis Spectrophotometer (Thermo Scientific).

For random barcode probes, a chemically-synthesized probe was purchased with a $5^{\prime}$ extension representing the complement of the primer-binding portion. Several thymine nucleotides were replaced with uracil in this region. The probe contained random nucleotides for the competitor side of the probe (as in Supplementary Fig. 2b) and ended with the $3^{\prime}$ palindromic domain. This domain was subsequently extended with Bst large fragment polymerase (NEB M0275), followed by USER-mediated cleavage (Uracil-Specific Excision Reagent, NEB, $\mathrm{M} 5505)$ of the $5^{\prime}$ uracil-containing domains and polyacrylamide gel purification.

NUPACK simulations. Expected reaction products at thermodynamic equilibrium were predicted using NUPACK ${ }^{32}$ web-based software (nupack.org), analysis option. Specified options were DNA nucleic acid type, $37^{\circ} \mathrm{C}$ temperature, $10 \mathrm{nM}$ reactants, with maximum complex size of two. Advanced options $0.05 \mathrm{mM} \mathrm{Na}^{+}$, $0.002 \mathrm{mM} \mathrm{Mg}^{2+}$, and all dangle treatments. Pair probability results were downloaded in text form and replotted using custom software (Wolfram Mathematica). See Supplementary Fig. 1.

APR recording. Recording in solution for most experiments was carried out as follows. Probe strands were bound to streptavidin, as above. A mixture of $100 \mu \mathrm{M}$ each dNTP (NEB), $1 \times$ ThemoPol Buffer (NEB, N0447), and $0.8 \mathrm{U} / \mu \mathrm{l}$ Bst large fragment polymerase, $200-400 \mathrm{nM}$ Cy5-labeled primer (IDT), and probe/streptavidin mix (per above) at $10 \mathrm{nM}$ final streptavidin concentration was prepared at $4^{\circ}$ $\mathrm{C}$ at a volume of $10 \mu \mathrm{l}$ per reaction. The temperature was raised to $37^{\circ} \mathrm{C}$ for 120 min (or other reaction time), followed by $80^{\circ} \mathrm{C}$ heat inactivation for $20 \mathrm{~min}$. The fastest reliable recording conditions found (specifically for newly optimized probe and conditions of Fig. 3b) utilize the iso- $\mathrm{dC} / \mathrm{dG}$ probes, $100 \mathrm{nM}$ primer (with phosphorothioate bond, IDT), $1 \times$ Bsm buffer, $1.2 \mathrm{U} / \mu$ l total Bsm (Thermo Fisher, EP0691), $150 \mu \mathrm{M}$ dNTPs, and an additional $4 \mathrm{mM} \mathrm{MgSO}_{4}$.

For recording reactions on origami, flow chambers were created by attaching a freshly cleaved mica (Ted Pella) piece to a channel slide system (ibidi, Sticky-Slide VI0.4, Cat. No. 80608), yielding a $30 \mu \mathrm{l}$ chamber volume. After washing the chamber three times with $60 \mu \mathrm{l}$ of TAE/Mg buffer by adding the buffer to one reservoir (inlet) and subsequently taking out the same volume from the other side (outlet), a $30 \mu \mathrm{l}$ solution containing origami at $50 \mathrm{pM}$ concentration was introduced to the chamber. (In the first washing round, $\sim 30 \mu \mathrm{l}$ of buffer occupies the chamber and only $\sim 30 \mu \mathrm{l}$ of extra buffer comes out.) After $30 \mathrm{~min}$ of incubation, unbound origami and extra staple strands were removed by washing three times with $60 \mu \mathrm{l}$ of fresh TAE/Mg buffer $(40 \mathrm{mM}$ Tris acetate, $1 \mathrm{mM}$ EDTA buffer supplemented with $12.5 \mathrm{mM}$ magnesium acetate) and three times with $60 \mu \mathrm{l}$ of $1 \times$ ThermoPol buffer (NEB, Cat. No. B9004S, 10x; $1 \times$ contains $20 \mathrm{mM}$ Tris$\mathrm{HCl}, 10 \mathrm{mM}\left(\mathrm{NH}_{4}\right)_{2} \mathrm{SO}_{4}, 10 \mathrm{mM} \mathrm{KCl}, 2 \mathrm{mM} \mathrm{MgSO}_{4}, 0.1 \%$ Triton X-100). To the chamber, a $40 \mu \mathrm{l}$ solution containing 0.8 units/ $\mu \mathrm{l}$ of Bst polymerase (NEB, Cat. No. M0275S), $100 \mu \mathrm{M}$ dNTP (NEB, Cat. No. N0447S), and the relevant primer mixes typically at $100 \mathrm{nM}$ each, in $1 \times$ ThermoPol reaction buffer, was added and incubated for $1 \mathrm{~h}$ at room temperature. After the recording reaction, the supernatant solution containing produced records was collected and the polymerase was heat inactivated by incubating the solution at $80^{\circ} \mathrm{C}$ for $20 \mathrm{~min}$. For samples used in the geometry studies (three-point and hexagonal grid patterns) and the state change study, before quenching the reaction, extra recording primers contained in the product solution were removed by mixing the solution with Exonuclease I (NEB, Cat. No. M0293S) at 9:1 volume ratio and incubating for $20 \mathrm{~min}$ at $37^{\circ} \mathrm{C}$. This minimizes any false-positive PCR amplification by the recording primers.

State change recordings of Fig. 6 were carried out following the standard origami procedure described just above, except that the product solutions were collected after first adding $40 \mu \mathrm{l}$ of buffer into the inlet, to prevent drying of the chamber between rounds. After the first and second state recording rounds, the chamber was washed six times with $1 \times$ ThermoPol buffer before the wash samples were collected, followed by three times with TAE/Mg. For deactivating or reactivating the probe $\mathrm{P} 3$, a $40 \mu \mathrm{l}$ solution containing the deactivator strand at $10 \mu \mathrm{M}$ or the reactivator strand at $0.1 \mu \mathrm{M}$ respectively in $\mathrm{TAE} / \mathrm{Mg}$ was added and incubated for $30 \mathrm{~min}$ at room temperature, after which the chamber was washed four times with TAE/Mg and four times with $1 \times$ ThermoPol buffer before each recording reaction.

For the 7-point hexagonal grid geometry recordings of Fig. 5, the experimental condition is similar to the ones for stage change recording except that the annealing temperature for PCR amplification was to $52^{\circ} \mathrm{C}$, with $200 \mathrm{nM}$ of each primer sequence used in the PCR mixture.

Electrophoresis. Gels $(8 \times 8 \mathrm{~cm})$ for denaturing polyacrylamide gel electrophoresis (PAGE) were cast in house at $8 \%$ acrylamide (J.T.Baker), with $7 \mathrm{M}$ urea (Sigma) and $1 \times$ TAE ( $40 \mathrm{mM}$ Tris, $20 \mathrm{mM}$ acetic acid, $1 \mathrm{mM}$ EDTA) buffer (GrowCells com), in plastic cassettes (Life Technologies), and run for $30-35 \mathrm{~min}$ at $200 \mathrm{~V}$ and $65^{\circ} \mathrm{C}$ in $1 \times$ TAE buffer. Gels were then removed from cassettes, stained in $1 \times$ SybrGold (Life Technologies) for $15 \mathrm{~min}$, and imaged with a Typhoon scanner (General Electric). All ladders shown are 10-300 nt (Thermo Scientific, Cat. No. SM1213).

DNA origami preparation. The design and folding protocols of the $2 \mathrm{D}$ rectangular origami were adapted from the original rectangle ${ }^{3}$ and the twist-corrected version $^{13,37}$. Single-stranded M13mp18 DNA (scaffold strand) was purchased from Bayou Biolabs (Catalog P-107) and staple strands were obtained from IDT. Staple strands were purchased and used unpurified, except the staples with probe extension at the $5^{\prime}$ end of the staples, which were purchased PAGE-purified. Scaffold and staples were mixed together at target concentrations of 5 and $40 \mathrm{nM}$, respectively, in TAE buffer with $12.5 \mathrm{mM}$ magnesium acetate. For origami with click-coupled probes, the probe strands were added at 1.2-fold the concentration of the staple strands containing the anchor. For origami folding, the mixtures were kept at $90^{\circ} \mathrm{C}$ for $5 \mathrm{~min}$ and annealed from 90 to $60^{\circ} \mathrm{C}$ over the course of $30 \mathrm{~min}$, then from 60 to $45^{\circ} \mathrm{C}$ over the course of $90 \mathrm{~min}$, and finally from 45 to $25^{\circ} \mathrm{C}$ over the course of $20 \mathrm{~min}$. Folded origami solutions were used without further purification except where otherwise stated. See Supplementary Fig. 5 for geometry and layout and Supplementary Table 1 for staple sequences.

Record PCR amplification. PCR amplification utilized 1× VentR (exo-) polymerase in $1 \times$ ThermoPol Buffer (NEB), for 20 cycles, in $20 \mu \mathrm{l}$ reactions. Primers were initially present at $0.5 \mu \mathrm{M}$, and dNTP mix (NEB) at $100 \mu \mathrm{M}$ each. Temperature cycling included a $95^{\circ} \mathrm{C}$ melt for $2 \mathrm{~min}, 20$ cycles (varies) of $95^{\circ} \mathrm{C}$ for $20 \mathrm{~s}$ denaturing, $56^{\circ} \mathrm{C}$ for $45 \mathrm{~s}$ annealing, $72{ }^{\circ} \mathrm{C}$ for $15 \mathrm{~s}$ extension, and a final $72^{\circ} \mathrm{C}$ extension for $3 \mathrm{~min}$.

Data availability. The data that support the findings of this study are available from the authors on reasonable request.

Received: 10 August 2016 Accepted: 27 June 2017 Published online: 25 September 2017

\section{References}

1. Paul, R. K. et al. Synthesis of a pillared graphene nanostructure: a counterpart of three-dimensional carbon architectures. Small 6, 2309-2313 (2010).

2. Ming, T. et al. Ordered gold nanostructure assemblies formed by droplet evaporation. Angew. Chem. Int. Ed. Engl. 120, 9831-9836 (2008).

3. Rothemund, P. W. K. Folding DNA to create nanoscale shapes and patterns. Nature 440, 297-302 (2006).

4. Wei, B., Dai, M. \& Yin, P. Complex shapes self-assembled from modular molecular components. Nature 485, 623-626 (2012).

5. Ke, Y., Ong, L., Shih, W. M. \& Yin, P. Three-dimensional structures selfassembled from DNA bricks. Science 338, 1177-1183 (2012).

6. Alber, F. et al. The molecular architecture of the nuclear pore complex. Nature 450, 695-701 (2007).

7. Zaidel-Bar, R., Itzkovitz, S., Ma'ayan, A., Iyengar, R. \& Geiger, B. Functional atlas of the integrin adhesome. Nat. Cell. Biol. 9, 858-867 (2007).

8. Suderman, R. \& Deeds, E. J. Machines vs. ensembles: effective MAPK signaling through heterogeneous sets of protein complexes. PLoS Comput. Biol. 9, e1003278 (2013).

9. Sengupta, P. et al. Probing protein heterogeneity in the plasma membrane using PALM and pair correlation analysis. Nat. Methods 8, 969-975 (2011)

10. Ebong, I.-o et al. Heterogeneity and dynamics in the assembly of the heat shock protein 90 chaperone complexes. Proc. Natl. Acad. Sci. USA 108, 17939-17944 (2011).

11. Jungmann, R. et al. Quantitative super-resolution imaging with qPAINT. Nat. Methods 13, 439-442 (2016)

12. Barabasi, A.-L. \& Oltvai, Z. N. Network biology: understanding the cell's functional organization. Nat. Rev. Genet. 5, 101-113 (2004). 
13. Jungmann, R. et al. Multiplexed 3D cellular super-resolution imaging with DNA-PAINT and exchange-PAINT. Nat. Methods 11, 313-318 (2014).

14. Svitkina, T. M. \& Borisy, G. G. Arp2/3 complex and actin depolymerizing factor/cofilin in dendritic organization and treadmilling of actin filament array in lamellipodia. J. Cell. Biol. 145, 1009-1026 (1999).

15. Hinterdorfer, P. \& Dufrêne, Y. F. Detection and localization of single molecular recognition events using atomic force microscopy. Nat. Methods 3, 347-355 (2006).

16. Kanchanawong, P. et al. Nanoscale architecture of integrin-based cell adhesions. Nature. 468, 580-584 (2010).

17. Dai, M., Jungmann, R. \& Yin, P. Optical imaging of individual biomolecules in densely packed clusters. Nat. Nanotechnol. 11, 798-807 (2016).

18. Santangelo, P., Nitin, N. \& Bao, G. Nanostructured probes for RNA detection in living cells. Ann. Biomed. Eng. 34, 39-50 (2006).

19. Shih, W. M., Gryczynski, Z., Lakowicz, J. R. \& Spudich, J. A. A FRET-based sensor reveals ATP hydrolysis-induced large conformational changes and three distinct states of the molecular motor myosin. Cell 102, 683-694 (2000).

20. Stelzl, U. et al. A human protein-protein interaction network: a resource for annotating the proteome. Cell 122, 957-968 (2005).

21. $\mathrm{Yu}, \mathrm{H}$. et al. High-quality binary protein interaction map of the yeast interactome network. Science 322, 104-110 (2008).

22. Ito, T. et al. A comprehensive two-hybrid analysis to explore the yeast protein interactome. Proc. Natl. Acad. Sci. USA 98, 4569-4574 (2001).

23. Tarassov, K. et al. An in vivo map of the yeast protein interactome. Science 320, 1465-1470 (2008).

24. Gavin, A.-C. et al. Functional organization of the yeast proteome by systematic analysis of protein complexes. Nature 415, 141-147 (2002).

25. Gao, J. et al. A protein-protein interaction network of transcription factors acting during liver cell proliferation. Genomics 91, 347-355 (2008).

26. Fredriksson, S. et al. Protein detection using proximity-dependent DNA ligation assays. Nat. Biotechnol. 20, 473-477 (2002).

27. Assarsson, E. et al. Homogenous 96-plex PEA immunoassay exhibiting high sensitivity, specificity, and excellent scalability. PLoS ONE 9, e95192 (2014).

28. Klein, A. M. et al. Droplet barcoding for single-cell transcriptomics applied to embryonic stem cells. Cell 161, 1187-1201 (2015).

29. Yurke, B., Turberfield, A., Mills, Jr A., Simmel, F. \& Neumann, J. A DNA-fuelled molecular machine made of DNA. Nature 406, 605-608 (2000).

30. Radding, C., Beattie, K., Holloman, W. \& Wiegand, R. Uptake of homologous single-stranded fragments by superhelical DNA. IV. branch migration. J. Mol. Biol. 166, 825-839 (1977).

31. Zhang, D. Y. \& Winfree, E. Control of DNA strand displacement kinetics using toehold exchange. J. Am. Chem. Soc. 131, 17303 (2009).

32. Zadeh, J. N. et al. NUPACK: analysis and design of nucleic acid systems. J. Comput. Chem. 32, 170-173 (2011).

33. SantaLucia, J. \& Hicks, D. The thermodynamics of DNA structural motifs. Annu. Rev. Biophys. Biomol. Struct. 33, 415-440 (2004).

34. Smith, S. B., Yujia, C. \& Bustamante, C. Overstretching B-DNA: the elastic response of individual double-stranded and single-stranded DNA groups. Science 271, 795-799 (1996).

35. Aubert, N., Rondelez, Y., Fujii, T. \& Hagiya, M. Enforcing logical delays in DNA computing systems. Nat. Comput. 13, 559-572 (2014).

36. Shiroguchi, K., Jia, T. Z., Sims, P. A. \& Xie, X. S. Digital RNA sequencing minimizes sequence-dependent bias and amplification noise with optimized single-molecule barcodes. Proc. Natl. Acad. Sci. 109, 1347-1352 (2012).

37. Woo, S. \& Rothemund, P. W. K. Programmable molecular recognition based on the geometry of DNA nanostructures. Nat. Chem. 3, 620-627 (2011).

38. Rivetti, C., Guthold, M. \& Bustamante, C. Scanning force microscopy of DNA deposited onto mica: equilibration versus kinetic trapping studied by statistical polymer chain analysis. J. Mol. Biol. 264, 919-932 (1996).
39. Murphy, M., Rasnik, I., Cheng, W., Lohman, T. M. \& Ha, T. Probing singlestranded DNA conformational flexibility using fluorescence spectroscopy. Biophys. J. 86, 2530-2537 (2004).

\section{Acknowledgements}

We thank Jocelyn Kishi, Nikhil Gopalkrishnan, Reza Kalhor, and Mitchell Zakin for fruitful discussions. This work is supported by a National Institutes of Health (NIH) Director's New Innovator Award (1DP2OD007292), an NIH Transformative Research Award (1R01EB018659), an Office of Naval Research (ONR) Young Investigator Program Award (N000141110914), ONR grants (N00014-13-1-0593, N00014-14-1-0610, N00014-16-1-2182, and N00014-16-1-2410), a National Science Foundation (NSF) Faculty Early Career Development Award (CCF1054898), an NSF Expedition in Computing Award (CCF1317291), the Wyss Institute for Biologically Inspired Engineering Molecular Robotics Initiative, and a Wyss Institute Faculty Startup Fund to P.Y. T.E.S. and X.C. acknowledge support from the Jane Coffin Childs Postdoctoral Fellowship, and S.W. is a HHMI Fellow of the Damon Runyon Cancer Research Foundation (DRG-220314).

\section{Author contributions}

T.E.S. conceived of and designed the study, designed and developed the APR mechanism, designed and performed the experiments, analyzed the data, and wrote the manuscript. S.W. designed and performed the experiments, analyzed the data, and wrote the manuscript. F.X. designed and performed the experiments and analyzed the data. X.C. designed and performed the experiments, developed the APR mechanism, and analyzed the data. P.Y. conceived of and supervised the study, developed the APR mechanism, analyzed the data, and wrote the manuscript. All authors reviewed and approved the manuscript.

\section{Additional information}

Supplementary Information accompanies this paper at doi:10.1038/s41467-017-00542-3.

Competing interests: T.E.S., X.C., and P.Y. have filed a provisional patent based on this work. P.Y. is a co-founder of Ultivue, Inc. and NuProbe Global. The remaining authors declare no competing financial interests.

Reprints and permission information is available online at http://npg.nature.com/ reprintsandpermissions/

Publisher's note: Springer Nature remains neutral with regard to jurisdictional claims in published maps and institutional affiliations.

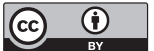

Open Access This article is licensed under a Creative Commons Attribution 4.0 International License, which permits use, sharing, adaptation, distribution and reproduction in any medium or format, as long as you give appropriate credit to the original author(s) and the source, provide a link to the Creative Commons license, and indicate if changes were made. The images or other third party material in this article are included in the article's Creative Commons license, unless indicated otherwise in a credit line to the material. If material is not included in the article's Creative Commons license and your intended use is not permitted by statutory regulation or exceeds the permitted use, you will need to obtain permission directly from the copyright holder. To view a copy of this license, visit http://creativecommons.org/ licenses/by/4.0/.

(C) The Author(s) 2017 